\title{
Hartogs' Phenomena for Microfunctions with Holomorphic Parameters
}

\author{
By \\ Otto Liess, Yasunori OKADA** and Nobuyuki Tose***
}

\section{$\S 1$. Statement of the Results}

1. Hyperfunctions and microfunctions with holomorphic parameters have been considered in classical microlocalization and they play an important role in second microlocalization. Our main aim in this paper is to prove a microlocal variant of the Malgrange-Zerner theorem in hyperfunctions with holomorphic parameters. See Theorem 2.1 below and for a classical version of MalgrangeZerner type theorem, see H. Komatsu [6]. The interest in the theorem lies in the fact that it shows that microfunctions with holomorphic parameters are pointwise determined. The corresponding theorem for hyperfunctions with holomorphic parameters has been obtained before by K. Kataoka and independently by T. Oshima; actually their result is somewhat stronger. They studied the problem for hyperfunctions with real analytic parameters (see Corollary 1.2 and Theorem 2.8). It was not published, but it was included with proof as Theorem 4.4.7' in A. Kaneko [3].

Before we state the main result, let us fix the situation where we work:

Let $M^{\prime}$ be a real analytic manifold with complexification $X^{\prime}$ and let $X^{\prime \prime}$ be a complex manifold. Local coordinates of $M^{\prime}, X^{\prime}$, and $X^{\prime \prime}$ are denoted by $x^{\prime}, z^{\prime}$, and $z^{\prime \prime}$ respectively. We consider the embedding $N:=M^{\prime} \times X^{\prime \prime} \hookrightarrow X:=$ $X^{\prime} \times X^{\prime \prime}$ and identify the conormal bundle $T_{N}^{*} X$ along $N$ with $T_{M^{\prime}}^{*} X^{\prime} \times X^{\prime \prime}$.

Communicated by T. Kawai, June 6, 2000. Revised November 8, 2000.

2000 Mathematics Subject Classification(s): 32A45, 32D99.

* Department of Mathematics, Università degli Studi di Bologna, Piazza Porta San Donato 5, Bologna 40127, Italy.

e-mail: liess@dm.unibo.it

** Department of Mathematics and Informatics, Faculty of Science, Chiba University, Yayoi 1-33, Inage-ku, Chiba 263-8522, Japan. e-mail: okada@math.s.chiba-u.ac.jp

*** Mathematics, Hiyoshi Campus, Keio University, Hiyoshi 4-1-1, Kohoku, Yokohama 2238521, Japan.

e-mail: tose@math.hc.keio.ac.jp 
The canonical projection from $T_{N}^{*} X$ to $N\left(\operatorname{resp} . T_{M^{\prime}}^{*} X^{\prime}\right.$ to $\left.M^{\prime}\right)$ is denoted by $\pi_{N}$ (resp. $\pi_{M^{\prime}}$ ). We introduce the sheaf $\mathcal{C O}_{N}$ of microfunctions with holomorphic parameter $z^{\prime \prime}$ on $T_{N}^{*} X$ by

$$
\mathcal{C} \mathcal{O}_{N}:=\mu_{N}\left(\mathcal{O}_{X}\right) \otimes o r_{N / X}\left[\operatorname{dim}_{\mathbb{R}} M^{\prime}\right]
$$

and the sheaf $\mathcal{B O}_{N}$ of hyperfunctions with holomorphic parameter $z^{\prime \prime}$ on $N$ by

$$
\mathcal{B O}_{N}:=\left.\mathcal{C O}_{N}\right|_{N}
$$

Here $\mathcal{O}_{X}$ denotes the sheaf of holomorphic functions on $X, \mu_{N}$ Sato's microlocalization functor along $N$, and $o r_{N / X}$ the relative orientation sheaf. For any fixed point $\dot{z}^{\prime \prime} \in X^{\prime \prime}$, we can define the restriction morphisms

$$
\left.\mathcal{B O}_{N}\right|_{\left\{z^{\prime \prime}=\dot{z}^{\prime \prime}\right\}} \rightarrow \mathcal{B}_{M^{\prime}},\left.\quad u \mapsto u\right|_{z^{\prime \prime}=\dot{z}^{\prime \prime}}
$$

and

$$
\left.\mathcal{C O} \mathcal{O}_{N}\right|_{\left\{z^{\prime \prime}=\dot{z}^{\prime \prime}\right\}} \rightarrow \mathcal{C}_{M^{\prime}},\left.\quad u \mapsto u\right|_{z^{\prime \prime}=\dot{z}^{\prime \prime}}
$$

under the identifications $M^{\prime} \times\left\{\dot{z}^{\prime \prime}\right\} \simeq M^{\prime}$ and $T_{M^{\prime}}^{*} X^{\prime} \times\left\{\dot{z}^{\prime \prime}\right\} \simeq T_{M^{\prime}}^{*} X^{\prime}$. Here we denote by $\mathcal{B}_{M^{\prime}}$ the sheaf of hyperfunctions on $M^{\prime}$ and by $\mathcal{C}_{M^{\prime}}$ that of microfunctions on $T_{M^{\prime}}^{*} X^{\prime}$.

Now we state our main theorem:

Theorem 1.1. Let $\dot{q}^{\prime} \in T_{M^{\prime}}^{*} X^{\prime}$ be a point and $U^{\prime \prime} \subset X^{\prime \prime}$ an open subset. The map

$$
\mathcal{C} \mathcal{O}_{N}\left(\left\{\dot{q}^{\prime}\right\} \times U^{\prime \prime}\right) \rightarrow \prod_{\dot{z}^{\prime \prime} \in U^{\prime \prime}} \mathcal{C}_{M^{\prime}, \dot{q}^{\prime}}
$$

defined by $u \mapsto\left(\left.u\right|_{z^{\prime \prime}=\dot{z}^{\prime \prime}}\right)_{\dot{z}^{\prime \prime} \in U^{\prime \prime}}$ is injective.

We give explicitly two corollaries of Theorem 1.1.

Corollary 1.2. Let $\dot{x}^{\prime} \in M^{\prime}$ be a point, $U^{\prime \prime} \subset X^{\prime \prime}$ an open subset and $u \in \mathcal{B O}_{N}\left(\left\{\dot{x}^{\prime}\right\} \times U^{\prime \prime}\right)$ a hyperfunction with holomorphic parameter $z^{\prime \prime}$ defined in a neighborhood of $\left\{\dot{x}^{\prime}\right\} \times U^{\prime \prime}$. Assume that for any $\dot{z}^{\prime \prime} \in U^{\prime \prime}$, the restriction $\left.u\right|_{z=\dot{z}^{\prime \prime}}=0$ at $\dot{x}^{\prime}$. Then $u=0$ in a neighborhood of $\left\{\dot{x}^{\prime}\right\} \times U^{\prime \prime}$.

Corollary 1.3. Let $\Omega^{\prime} \subset M^{\prime}$ and $U^{\prime \prime} \subset X^{\prime \prime}$ be two open subsets and $u \in \mathcal{B O}_{N}\left(\Omega^{\prime} \times U^{\prime \prime}\right)$ a hyperfunction with holomorphic parameter. Assume that for any $\dot{z}^{\prime \prime} \in U^{\prime \prime}$, the restriction $\left.u\right|_{z=\dot{z}^{\prime \prime}} \in \mathcal{B}_{M^{\prime}}\left(\Omega^{\prime}\right)$ is real analytic. Then $u$ itself is an analytic function on $\Omega^{\prime} \times U^{\prime \prime}$, i.e., there exist a neighborhood $\tilde{U} \subset X$ of $\Omega^{\prime} \times U^{\prime \prime}$ and a holomorphic function $f \in \mathcal{O}_{X}(\tilde{U})$ with $u=\left.f\right|_{\Omega^{\prime} \times U^{\prime \prime}}$. 
We remark that Corollary 1.2 can be obtained from a result due to Kataoka and Oshima given in Theorem 2.8, in which $u\left(x^{\prime}, x^{\prime \prime}\right)$ is assumed to have $x^{\prime \prime}$ as real analytic parameters.

Let us consider moreover the case where $X^{\prime \prime}$ is the complexification of some real analytic manifold $M^{\prime \prime}$. In this situation, we give a stronger result:

Theorem 1.4. Let $\dot{q}^{\prime} \in T_{M^{\prime}}^{*} X^{\prime}$ be a point and $U^{\prime \prime} \subset X^{\prime \prime}$ a connected open set. Assume that $M^{\prime \prime} \cap U^{\prime \prime}$ is non-empty. Then the map

$$
\mathcal{C} \mathcal{O}_{N}\left(\left\{\dot{q}^{\prime}\right\} \times U^{\prime \prime}\right) \rightarrow \prod_{\dot{x}^{\prime \prime} \in M^{\prime \prime} \cap U^{\prime \prime}} \mathcal{C}_{M^{\prime}, \dot{q}^{\prime}}
$$

defined by $u \mapsto\left(\left.u\right|_{z^{\prime \prime}=\dot{x}^{\prime \prime}}\right)_{\dot{x}^{\prime \prime} \in M^{\prime \prime} \cap U^{\prime \prime}}$ is injective.

Note that since we can argue locally, Theorem 1.1 will be a consequence of Theorem 1.4.

2. Let us next consider the sequence of embeddings

$$
M:=M^{\prime} \times M^{\prime \prime} \hookrightarrow N \hookrightarrow X,
$$

which defines the sheaf

$$
\mathcal{A}_{\Sigma}^{2}:=\left.\mathcal{C O}_{N}\right|_{\Sigma}
$$

of second analytic functions defined on the real regular involutive submanifold

$$
\Sigma:=T_{M}^{*} X \times_{T^{*} X} T_{N}^{*} X .
$$

It is again a consequence of Theorem 1.4 that the sections of $\mathcal{A}_{\Sigma}^{2}$ are determined pointwisely:

Corollary 1.5. Let $\dot{q}^{\prime} \in T_{M^{\prime}}^{*} X^{\prime}$ be a point, $\Omega^{\prime \prime} \subset M^{\prime \prime}$ an open subset, and $u \in \mathcal{A}_{\Sigma}^{2}\left(\left\{\dot{q}^{\prime}\right\} \times \Omega^{\prime \prime}\right)$ a second analytic function. Assume that $\left.u\right|_{x^{\prime \prime}=\dot{x}^{\prime \prime}}=0$ for any fixed $\dot{x}^{\prime \prime} \in \Omega^{\prime \prime}$. Then $u=0$.

The following particular case of Theorem 1.4 will be the main intermediate step in the argument. We denote in it by $\dot{T}_{M^{\prime}}^{*} X^{\prime}=T_{M^{\prime}}^{*} X^{\prime} \backslash M^{\prime}$ the conormal bundle to $M^{\prime}$ with the zero section removed and by $\dot{\pi}_{M^{\prime}}$ the canonical projection from $\dot{T}_{M^{\prime}}^{*} X^{\prime}$ to $M^{\prime}$.

Theorem 1.6. Let $\Omega^{\prime} \subset M^{\prime}$ be an open subset, $U^{\prime \prime} \subset X^{\prime \prime}$ a connected open subset with $U^{\prime \prime} \cap M^{\prime \prime} \neq \emptyset$, and $Z \subset \dot{\pi}_{M^{\prime}}^{-1}\left(\Omega^{\prime}\right)$ a closed conic subset such 
that for each base point $\dot{x}^{\prime} \in \Omega^{\prime}$ the intersection $Z \cap \dot{\pi}_{M^{\prime}}^{-1}\left(\dot{x}^{\prime}\right)$ consists of only one direction. Also consider a fixed point $\dot{q}^{\prime}$ in $Z$.

Assume that a section $u \in \mathcal{C O}_{N}\left(\dot{\pi}_{M^{\prime}}^{-1}\left(\Omega^{\prime}\right) \times U^{\prime \prime}\right)$ satisfies the condition

$$
\operatorname{supp}(u) \subset Z \times U^{\prime \prime}
$$

and that $\left.u\right|_{z^{\prime \prime}=\dot{x}^{\prime \prime}}=0$ at $\dot{q}^{\prime}$ for any $\dot{x}^{\prime \prime} \in U^{\prime \prime} \cap M^{\prime \prime}$. Then we have $u=0$ in a neighborhood of $\left\{\dot{q}^{\prime}\right\} \times U^{\prime \prime}$.

\section{$\S 2$. Local Forms of the Main Results}

1. The theorems above in Section 1 are of a local nature. We may argue therefore in local coordinates and assume that $X^{\prime}=\mathbb{C}^{d}, X^{\prime \prime}=\mathbb{C}^{n-d}$ for some $d$ and $n$. We shall identify $X^{\prime} \times X^{\prime \prime}$ with $\mathbb{C}^{n}$ in a natural way: if $z=\left(z_{1}, \ldots, z_{n}\right)$ are the coordinates in $\mathbb{C}^{n}$, we write $z^{\prime}$ for $\left(z_{1}, \ldots, z_{d}\right)$ and $z^{\prime \prime}$ for $\left(z_{d+1}, \ldots, z_{n}\right)$. Thus, $z=\left(z^{\prime}, z^{\prime \prime}\right)$ and $X^{\prime}=\left\{z \in \mathbb{C}^{n} ; z^{\prime \prime}=0\right\}, X^{\prime \prime}=\left\{z \in \mathbb{C}^{n} ; z^{\prime}=0\right\}$. We denote $M=\{z \in X ; \operatorname{Im} z=0\}=\mathbb{R}^{n}$, regarded as a real analytic submanifold in $\mathbb{C}^{n}$ and consider its partial complexification $N=\left\{z \in X ; \operatorname{Im} z^{\prime}=0\right\}=$ $\mathbb{R}^{d} \times \mathbb{C}^{n-d}$. Variables in $M$ shall be written as $x=\left(x^{\prime}, x^{\prime \prime}\right), x^{\prime}=\left(x_{1}, \ldots, x_{d}\right)$, $x^{\prime \prime}=\left(x_{d+1}, \ldots, x_{n}\right)$. Coordinates of $T_{N}^{*} X=T_{\mathbb{R}^{d}}^{*} \mathbb{C}^{d} \times \mathbb{C}^{n-d}$ are denoted by $\left(x^{\prime}, z^{\prime \prime} ; \xi^{\prime} \cdot d x^{\prime}\right)$ or $\left(x^{\prime}, z^{\prime \prime} ; \xi^{\prime}\right)$ under the identification $T_{\mathbb{R}^{d}}^{*} \mathbb{C}^{d} \simeq \sqrt{-1} T^{*} \mathbb{R}^{d} \simeq$ $T^{*} \mathbb{R}^{d}$.

The sheaf $\mathcal{B O}_{N}$ is in this situation isomorphic to the sheaf associated with the following presheaf $\mathcal{F}$ : for open sets $\Omega^{\prime} \subset \mathbb{R}^{d}$ and $U^{\prime \prime} \subset \mathbb{C}^{n-d}$,

$$
\mathcal{F}\left(\Omega^{\prime} \times U^{\prime \prime}\right)=\bigsqcup_{U^{\prime}, G_{1}^{\prime}, \ldots, G_{s}^{\prime}}\left\{\sum_{j=1}^{s} F_{j} ; F_{j} \in \mathcal{O}\left(\left\{z \in U^{\prime} \times U^{\prime \prime} ; \operatorname{Im} z^{\prime} \in G_{j}^{\prime}\right\}\right)\right\} / \sim
$$

where $U^{\prime}$ runs through the set of all open complex neighborhoods of $\Omega^{\prime}$ in $\mathbb{C}^{d}$, and $\left(G_{1}^{\prime}, \ldots, G_{s}^{\prime}\right)$ runs through all the finite collections of open convex cones in $\mathbb{R}^{d}$. Here $\sum_{j} F_{j} \sim 0$ means that there exist a complex neighborhood $\tilde{U}^{\prime}$ of $\Omega^{\prime}$ in $U^{\prime}$, open convex cones $\tilde{G}_{j}^{\prime} \subset \subset G_{j}^{\prime}(j=1,2, \ldots, s)$, and holomorphic functions $F_{j k} \in \mathcal{O}\left(\left\{z \in \tilde{U}^{\prime} \times U^{\prime \prime} ; \operatorname{Im} z^{\prime} \in \tilde{G}_{j}^{\prime}+\tilde{G}_{k}^{\prime}\right\}\right)(j, k=1,2, \ldots, s)$ satisfying $F_{j k}=-F_{k j}(\forall j, \forall k)$ and $F_{j}=\sum_{k=1}^{s} F_{j k}(j=1,2, \ldots, s)$. We denote the class of $F \in \mathcal{O}\left(\left\{z \in U^{\prime} \times U^{\prime \prime} ; \operatorname{Im} z^{\prime} \in G^{\prime}\right\}\right)$ in $\mathcal{B O}_{N}\left(\Omega^{\prime} \times U^{\prime \prime}\right)$ by $\dot{b}(F)$. Thus a section $u$ of $\mathcal{B O}_{N}$ locally admits a "boundary value representation"

$$
u=\sum_{j=1}^{s} \dot{b}\left(F_{j}\right)
$$


For any point $\dot{z}^{\prime \prime} \in \mathbb{C}^{n-d}$, the restriction map (1.3) is written as $u=\sum_{j} \dot{b}\left(F_{j}\right) \mapsto$ $\left.u\right|_{z^{\prime \prime}=\dot{z}^{\prime \prime}}=\sum_{j} b\left(\left.F_{j}\right|_{z^{\prime \prime}=\dot{z}^{\prime \prime}}\right)$. (If $F \in \mathcal{O}\left(\left\{z^{\prime} \in \mathbb{C}^{d} ; \operatorname{Re} z^{\prime} \in \Omega^{\prime}, \operatorname{Im} z^{\prime} \in G^{\prime}\right\}\right)$, we denote by $b(F)$, or by $F\left(x^{\prime}+\sqrt{-1} G^{\prime} 0\right)$, the hyperfunctional boundary value of $F$.)

For a section $u$ of $\mathcal{B O}_{N}$, we define the singular spectrum $\mathrm{WF}_{A}(u) \subset T_{N}^{*} X$ of $u$ as follows: a point $\left(\dot{x}^{\prime}, \dot{z}^{\prime \prime} ; \dot{\xi}^{\prime}\right) \in T_{N}^{*} X$ does not belong to $\mathrm{WF}_{A}(u)$ if and only if there exists a boundary value representation $u=\sum_{j} \dot{b}\left(F_{j}\right),\left(F_{j} \in\right.$ $\left.\mathcal{O}\left(\left\{z \in U^{\prime} \times U^{\prime \prime} ; \operatorname{Im} z^{\prime} \in G_{j}^{\prime}\right\}\right)\right)$ in a neighborhood of $\left(\dot{x}^{\prime}, \dot{z}^{\prime \prime}\right)$ so that $\dot{\xi}^{\prime} \notin G_{j}^{\prime \perp}$ for any $j$. (Cf. [3].) In view of this definition and of the definition of the singular spectrum for standard hyperfunctions, it is immediate that

$$
\mathrm{WF}_{A}\left(\left.u\right|_{z^{\prime \prime}=\dot{z}^{\prime \prime}}\right) \times\left\{\dot{z}^{\prime \prime}\right\} \subset \mathrm{WF}_{A}(u) \cap\left\{z^{\prime \prime}=\dot{z}^{\prime \prime}\right\}
$$

for any $\dot{z}^{\prime \prime}$. It is a consequence of our main result that the converse is also almost true: if $\left(\dot{x}^{\prime}, \dot{\xi}^{\prime}\right) \notin \mathrm{WF}_{A} u\left(\cdot, z^{\prime \prime}\right)$ for all complex $z^{\prime \prime}$ close to $\dot{z}^{\prime \prime}$, then $\left(\dot{x}^{\prime}, \dot{z}^{\prime \prime} ; \dot{\xi}^{\prime}\right) \notin \mathrm{WF}_{A} u$.

The sheaf $\mathcal{C O}_{N}$ is isomorphic to the sheaf associated with the following conical presheaf $\mathcal{G}$ : for any conical open subset $U$ in $T_{N}^{*} X$,

$$
\mathcal{G}(U):=\mathcal{B O}_{N}\left(\pi_{N}(U)\right) /\left\{u \in \mathcal{B O}_{N}\left(\pi_{N}(U)\right) ; \mathrm{WF}_{A}(u) \cap U=\emptyset\right\} .
$$

Let $\left(\dot{x}^{\prime} ; \dot{\xi}^{\prime}\right)$ be a fixed point in $T_{\mathbb{R}^{d}}^{*} \mathbb{C}^{d}$ and $U^{\prime \prime}$ an open subset in $\mathbb{C}^{n-d}$. A section $u$ of $\mathcal{C} \mathcal{O}_{N}$ defined in a neighborhood of $\left\{\left(\dot{x}^{\prime}, z^{\prime \prime} ; \dot{\xi}^{\prime}\right) ; z^{\prime \prime} \in U^{\prime \prime}\right\}$ admits, locally in the $z^{\prime \prime}$ variables for $z^{\prime \prime}$ near $\dot{z}^{\prime \prime}$, a boundary value representation

$$
u=\dot{b}(F), F \in \mathcal{O}\left(\left\{z \in \mathbb{C}^{n} ;\left|z^{\prime}-\dot{x}^{\prime}\right|<\varepsilon, \operatorname{Im} z^{\prime} \in G^{\prime},\left|z^{\prime \prime}-\dot{z}^{\prime \prime}\right|<\varepsilon\right\}\right)
$$

for a suitable $\varepsilon>0$ and a suitable open convex cone $G^{\prime} \subset \mathbb{R}^{d}$ with $\dot{\xi}^{\prime} \in G^{\perp}$. Using this representation, the restriction map (1.4) is written as $u=\dot{b}(F) \mapsto$ $\left.u\right|_{z^{\prime \prime}=\dot{z}^{\prime \prime}}=b\left(\left.F\right|_{z^{\prime \prime}=\dot{z}^{\prime \prime}}\right)$.

We also denote the space of germs of hyperfunctions at $0 \in \mathbb{R}^{d}$ by $\mathcal{B}_{0}$ and fix some vector $\dot{\xi}^{\prime} \in \dot{\mathbb{R}}^{d}=\mathbb{R}^{d} \backslash\{0\}$.

It is instructive to rewrite Theorem 1.1 in local variables and in terms of defining functions:

Theorem 2.1. Assume that $h \in \mathcal{O}\left(\left\{z \in \mathbb{C}^{n} ;\left|z^{\prime}\right|<\varepsilon, \operatorname{Im} z^{\prime} \in G^{\prime}, z^{\prime \prime} \in\right.\right.$ $\left.\left.\mathbb{C}^{n-d},\left|z^{\prime \prime}\right|<\delta\right\}\right)$ and denote by $u$ the hyperfunction with holomorphic parameters on $\left\{\left(x^{\prime}, z^{\prime \prime}\right) ;\left|x^{\prime}\right|<\varepsilon,\left|z^{\prime \prime}\right|<\delta\right\}$ associated with $h$. Also consider $\dot{\xi}^{\prime} \in G^{\prime} \perp$ and assume that $\left(0, \dot{\xi}^{\prime}\right) \notin \mathrm{WF}_{A} u\left(\cdot, z^{\prime \prime}\right)$, for any $z^{\prime \prime}$ with $\left|z^{\prime \prime}\right|<\delta$. Then there are $\varepsilon^{\prime}>0, \delta^{\prime}>0$, open convex cones $G_{1}^{\prime}, \ldots, G_{s}^{\prime}$ in $\mathbb{R}^{d}$ so that $\dot{\xi}^{\prime} \notin G_{j}^{\prime}{ }^{\perp}$ and 
holomorphic functions $h_{j}$ defined on $\left\{z \in \mathbb{C}^{n} ;\left|z^{\prime}\right|<\varepsilon^{\prime}, y^{\prime} \in G_{j}^{\prime},\left|z^{\prime \prime}\right|<\delta^{\prime}\right\}$ so that $u$ is for $\left|z^{\prime \prime}\right|<\delta^{\prime}$ equal to $\sum_{j=1}^{s} \dot{b}\left(h_{j}\right)$.

We also give a version of Theorem 1.6 in local coordinates:

Theorem 2.2. $\quad$ Let $G^{\prime} \subset \mathbb{R}^{d}$ be an open convex cone and $h$ a holomorphic function defined on $\left\{z \in \mathbb{C}^{n} ;\left|z^{\prime}\right|<\varepsilon, y^{\prime} \in G^{\prime},\left|z^{\prime \prime}\right|<\varepsilon\right\}$. Assume that for every $\dot{x}^{\prime \prime} \in \mathbb{R}^{n-d}$ with $\left|\dot{x}^{\prime \prime}\right|<\varepsilon$, the holomorphic function $h\left(\cdot, \dot{x}^{\prime \prime}\right)=\left.h\right|_{z^{\prime \prime}=\dot{x}^{\prime \prime}}$ defined on $\left\{z^{\prime} \in \mathbb{C}^{d} ;\left|z^{\prime}\right|<\varepsilon, y^{\prime} \in G^{\prime}\right\}$ extends holomorphically to a neighborhood of $0 \in \mathbb{C}^{d}$. Then $h$ extends holomorphically to a neighborhood of the set $\left\{z \in \mathbb{C}^{n} ; z^{\prime}=0,\left|z^{\prime \prime}\right|<\varepsilon\right\}$.

Remark 2.3. As a consequence of Corollary 1.2 it is possible to recast the definition of hyperfunctions (respectively microfunctions) with holomorphic parameters considered above as follows. Let again $U^{\prime \prime} \subset \mathbb{C}^{n-d}$ be some open subset. A function $h: U^{\prime \prime} \rightarrow \mathcal{B}_{0}$ is then a hyperfunction with holomorphic parameter $z^{\prime \prime}$ precisely if for any $\tilde{z}^{\prime \prime} \in U^{\prime \prime}$ there is an open neighborhood $\tilde{U}^{\prime \prime}=$ $\tilde{U}_{\tilde{z}^{\prime \prime}}$ of $\tilde{z}^{\prime \prime}, \varepsilon>0$, a finite collection of open convex cones $G_{j}^{\prime} \subset \mathbb{R}^{d}, j=1, \ldots, s$, and holomorphic functions $\tilde{h}_{j} \in \mathcal{O}\left(z ;\left|z^{\prime}\right|<\varepsilon, \operatorname{Im} z^{\prime} \in G_{j}^{\prime}, z^{\prime \prime} \in \tilde{U}^{\prime \prime}\right)$, so that for any $z^{\prime \prime} \in \tilde{U}^{\prime \prime}, h\left(z^{\prime \prime}\right)$ is equal to $\sum_{j=1}^{s} b\left(h_{j}\left(\cdot, z^{\prime \prime}\right)\right)$.

Likewise, a function $h: U^{\prime \prime} \rightarrow \mathcal{C}_{\left(0, \dot{\xi}^{\prime}\right)}\left(\mathcal{C}_{\left(0, \dot{\xi}^{\prime}\right)}\right.$ denotes here the set of germs of microfunctions at the point $\left.\left(0, \dot{\xi}^{\prime}\right)\right)$ will be a microfunction with holomorphic parameter $z^{\prime \prime}$ precisely if for any $\tilde{z}^{\prime \prime} \in U^{\prime \prime}$ there is an open neighborhood $\tilde{U}^{\prime \prime}=$ $\tilde{U}_{\tilde{z}^{\prime \prime}}$ of $\tilde{z}^{\prime \prime}, \varepsilon>0$, an open cone $G^{\prime} \subset \mathbb{R}^{d}$ which contains $\dot{\xi}^{\prime}$ and a holomorphic function $\tilde{h} \in \mathcal{O}\left(z ;\left|z^{\prime}\right|<\varepsilon, \operatorname{Im} z^{\prime} \in G^{\prime}, z^{\prime \prime} \in \tilde{U}^{\prime \prime}\right)$, so that for any $\dot{z}^{\prime \prime} \in \tilde{U}^{\prime \prime}$, $h\left(\dot{z}^{\prime \prime}\right)$ is the microfunction defined by the microfunctional boundary value of the holomorphic function $z^{\prime} \rightarrow \tilde{h}\left(z^{\prime}, \dot{z}^{\prime \prime}\right),\left|z^{\prime}\right|<\varepsilon, \operatorname{Im} z^{\prime} \in G^{\prime}$. We shall call $\tilde{h}$ a local defining function for $h$ (near $\tilde{z}^{\prime \prime}$ ). When we want to make the dependence of $\tilde{h}$ on $\tilde{z}^{\prime \prime}$ explicit, we shall occasionally write $\tilde{h}_{\tilde{z}^{\prime \prime}}$.

Remark 2.4. It is a significant fact that the local defining functions $\tilde{h}_{\tilde{z}^{\prime \prime}}$ associated with the various $\tilde{z}^{\prime \prime}$ do not always admit a common holomorphic extension for all $z^{\prime \prime} \in U^{\prime \prime}$. (I.e., in general there will exist no $f \in \mathcal{O}\left(z ;\left|z^{\prime}\right|<\right.$ $\left.\varepsilon, \operatorname{Im} z^{\prime} \in G^{\prime}, z^{\prime \prime} \in U^{\prime \prime}\right)$ with $\left.\left(0, \dot{\xi}^{\prime}\right) \notin \mathrm{WF}_{A}\left(b^{\prime}\left[f\left(\cdot, z^{\prime \prime}\right)-h\left(z^{\prime \prime}\right)\right]\right), \forall z^{\prime \prime} \in U^{\prime \prime}.\right)$

Remark 2.5. Let us consider Corollary 1.3 again. This corollary says that the real analyticity of $\left.u\left(x^{\prime}, z^{\prime \prime}\right)\right|_{z^{\prime \prime}=\dot{z}^{\prime \prime}}$ for each $\dot{z}^{\prime \prime}$ implies the real analyticity of $u$. It is important in this result that $z^{\prime \prime}$ is allowed to vary in an open set in $\mathbb{C}^{n}$. Indeed, there is no analogous result when we only have assumptions for $z^{\prime \prime}$ real. This is the content of the following 
Lemma 2.6. Let $u$ be the hyperfunction on $\mathbb{R}^{2}$ defined by

$$
u\left(x_{1}, x_{2}\right)=\frac{x_{2}}{x_{1}+i x_{2}^{2}+i 0} .
$$

Then

$$
\mathrm{WF}_{A}(u)=\{(0,0 ; 1,0)\}
$$

In particular the restrictions of $u$ with respect to the $x_{2}$ variable are well-defined. All these restrictions are real analytic (in one variable), but $u$ itself is not real analytic.

Proof. All statements are simple. Indeed, when $x_{1} \neq 0$ or $x_{2} \neq 0, u$ is obviously real analytic near $\left(x_{1}, x_{2}\right)$ and near $(0,0) u$ is the boundary value of the holomorphic function $h(z)=z_{2} /\left(z_{1}+i z_{2}^{2}\right)$ along the cone $G=\left\{y \in \mathbb{R}^{2} ; y_{1}>\right.$ $\left.\varepsilon\left|y_{2}\right|\right\}$, for any $\varepsilon$. This gives in particular $\mathrm{WF}_{A}(u) \subset\{(0,0 ; 1,0)\}$. The opposite inclusion is also clear since $u$ is not real analytic. As for the restrictions of $u$ to $x_{2}=\dot{x}_{2}$, (which exist in view of (2.6)) we did already mention that they are real analytic for $\dot{x}_{2} \neq 0$. In the remaining case, when we restrict to $x_{2}=0$, $u_{\mid x_{2}=0}$ vanishes identically, so it is trivially real analytic.

We remark that a similar example was already obtained in A. Kaneko [2].

We also give the following

Example 2.7. Consider a holomorphic function

$$
h\left(z_{1}, z_{2}\right)=\sum_{j=1}^{\infty} \frac{i\left(-i z_{2}\right)^{j}}{j^{j}\left(z_{1}+i\left(z_{2}^{2}+j^{-3 j}\right)\right)}
$$

on $\left\{\left(z_{1}, z_{2}\right) \in \mathbb{C}^{2} ; \operatorname{Im} z_{1}>\left(\operatorname{Im} z_{2}\right)^{2}-\left(\operatorname{Re} z_{2}\right)^{2}\right\}$. The boundary value $u\left(x_{1}, x_{2}\right)$ of $h$ satisfies

(1) for any $\dot{x}_{2}$ and any $k=0,1,2, \ldots,\left.\partial_{x_{2}}^{k} u\right|_{x_{2}=\dot{x}_{2}}$ is well-defined and real analytic,

(2) $\mathrm{WF}_{A}(u)=\{(0,0 ; 1,0)\}$.

In fact, the property (1) and the inclusion $\mathrm{WF}_{A}(u) \subset\{(0,0 ; 1,0)\}$ is easy. We can also show the opposite inclusion as follows. Note that at the point $\left(i y_{1}, i y_{2}\right)$ with $y_{1}>y_{2}^{2}$, the value $h\left(i y_{1}, i y_{2}\right)=\sum_{j=1}^{\infty} j^{-j} y_{2}^{j} /\left(y_{1}-y_{2}^{2}+j^{-3 j}\right)$ is 
positive real number and in particular at the point $z(k)=\left(i y_{1}(k), i y_{2}(k)\right)=$ $\left(i\left(k^{-2}+k^{-3 k}\right), i k^{-1}\right)$, we have

$$
\begin{aligned}
h(z(k)) & =h\left(i y_{1}(k), i y_{2}(k)\right)=\sum_{j=1}^{\infty} \frac{1}{(k j)^{j}\left(k^{-3 k}+j^{-3 j}\right)} \\
& >\left.\frac{1}{(k j)^{j}\left(k^{-3 k}+j^{-3 j}\right)}\right|_{j=k}=\frac{k^{k}}{2} .
\end{aligned}
$$

Since $\lim _{k} z(k)=(0,0)$, the estimate above shows that $h$ is not bounded in any neighborhood of the origin.

2. We give a remark.

As is included with proof as Theorem 4.4.7' in A. Kaneko [3], we have the following result due to Kataoka and Oshima about a property of hyperfunctions with real analytic parameters. To state it, we define the sheaf

$$
\mathcal{B} \mathcal{A}:=\mathcal{H}_{M}^{d}\left(\left.\mathcal{O}_{X}\right|_{X^{\prime} \times M^{\prime \prime}}\right),
$$

which was introduced by M. Sato [10]. We remark that M. Sato discussed about restriction of hyperfunctions in [10] with the aid of the sheaf $\mathcal{B A}$ before the notion of singular spectrum came to being. In this situation, we have

Theorem 2.8 (K. Kataoka and T. Oshima). Let $\Omega^{\prime}$ and $\Omega^{\prime \prime}$ be open subsets of $M^{\prime}$ and $M^{\prime \prime}$ respectively. Let $u\left(x^{\prime}, x^{\prime \prime}\right) \in \mathcal{B} \mathcal{A}\left(\Omega^{\prime} \times \Omega^{\prime \prime}\right)$ and assume that

$$
\left.u\left(x^{\prime}, x^{\prime \prime}\right)\right|_{x^{\prime \prime}=x_{0}^{\prime \prime}}=0
$$

for any $x_{0}^{\prime \prime} \in \Omega^{\prime \prime}$. Then it follows that $u=0$.

3. It follows from the above discussion that all results mentioned so far will be reduced to Theorem 2.2. We shall therefore start the part of "proofs" of this paper with the proof of this theorem: see Section 3. In Section 4 we shall then prove Theorem 1.6 and in Section 5 we shall then prove Theorem 1.4.

\section{§3. Proof of Theorem 2.2}

1. In this section, we give a proof of Theorem 2.2. The argument will be based on several tools: a characterization of extendability of holomorphic functions by duality, Baire's principle, a theorem of Hartogs' type, and the unique continuation property of singularities along holomorphic parameters. 
2. First we shall prove a very simple result on extendability of holomorphic functions. Before we can state the result we need to introduce some additional notations and conventions. We shall in fact denote by $B^{\prime}(\delta)$ the polydisc $\left\{z^{\prime} \in\right.$ $\left.\mathbb{C}^{d} ;\left|z_{j}\right|<\delta, \forall j\right\}$ in $\mathbb{C}^{d}$ and shall use, for subsets $U^{\prime}, V^{\prime} \subset \mathbb{C}^{d}$, the conventions:

$$
H_{U^{\prime}}\left(\zeta^{\prime}\right):=\sup _{z^{\prime} \in U^{\prime}} \operatorname{Re}\left(-i\left\langle z^{\prime}, \zeta^{\prime}\right\rangle\right), \quad U^{\prime}+V^{\prime}:=\left\{z^{\prime}+\tilde{z}^{\prime} ; z^{\prime} \in U^{\prime}, \tilde{z}^{\prime} \in V^{\prime}\right\} .
$$

It is immediate that $H_{U^{\prime}+B^{\prime}(\delta)}\left(\zeta^{\prime}\right)=H_{U^{\prime}}\left(\zeta^{\prime}\right)+\delta \sum_{j=1}^{d}\left|\zeta_{j}\right|$. Finally, if $U^{\prime} \subset \mathbb{C}^{d}$ is an open set, we denote by $\mathcal{O}_{\mathbb{C}^{d}}^{\prime}\left(U^{\prime}\right)$ the space of analytic functionals on $\mathcal{O}_{\mathbb{C}^{d}}\left(U^{\prime}\right)$. For simplicity we shall assume that $U^{\prime}$ is convex. It is well-known that analytic functionals $v$ in $\mathcal{O}_{\mathbb{C}^{d}}^{\prime}\left(U^{\prime}\right)$ are characterized by the fact their FourierBorel transform

$$
\zeta^{\prime} \rightarrow \hat{v}\left(\zeta^{\prime}\right)=\mathcal{F}(v)\left(\zeta^{\prime}\right)=v\left(\exp \left[-i\left\langle z^{\prime}, \zeta^{\prime}\right\rangle\right]\right)
$$

satisfies an estimate of form

$$
\left|\hat{v}\left(\zeta^{\prime}\right)\right| \leq c \exp \left[H_{Q}\left(\zeta^{\prime}\right)\right]
$$

for some constant $C$ and some compact set $Q \subset U^{\prime}$.

Theorem 3.1 (Holomorphic extensions and Duality). Let $U^{\prime} \subset \mathbb{C}^{d}$ be a bounded open convex domain and $h \in \mathcal{O}_{\mathbb{C}^{d}}\left(U^{\prime}\right)$ a holomorphic function defined on $U^{\prime}$. Then $h$ extends holomorphically to $U^{\prime}+B^{\prime}(\delta)$ if and only if for any $\delta^{\prime}$ with $0<\delta^{\prime}<\delta$ there exists a constant $c_{\delta^{\prime}}$ satisfying

$$
\forall v \in \mathcal{O}_{\mathbb{C}^{d}}^{\prime}\left(U^{\prime}\right),\left|\hat{v}\left(\zeta^{\prime}\right)\right| \leq \exp \left\{H_{U^{\prime}+B^{\prime}\left(\delta^{\prime}\right)}\left(\zeta^{\prime}\right)\right\} \Longrightarrow|v(h)| \leq c_{\delta^{\prime}}
$$

Theorem 3.1 is essentially well-known, but we give a proof to make this article self-contained. The argument is based on the following two propositions.

Proposition 3.2. Let $0<\delta<\delta^{\prime}$ be given and consider $f \in \mathcal{O}_{\mathbb{C}^{d}}\left(\mathbb{C}^{d}\right)$ such that

$$
\left|f\left(\zeta^{\prime}\right)\right| \leq \exp \left[\delta\left|\zeta^{\prime}\right|\right]
$$

Denote by $f_{j}\left(\zeta^{\prime}\right)=\sum_{|\alpha| \leq j}\left(\partial / \partial \zeta^{\prime}\right)^{\alpha} f(0) \zeta^{\prime \alpha} / \alpha$ ! the Taylor-polynomial of $f$ of order $j$ calculated at 0 . Then

$$
\sup _{\zeta^{\prime}}\left|f\left(\zeta^{\prime}\right)-f_{j}\left(\zeta^{\prime}\right)\right| \exp \left[-\delta^{\prime}\left|\zeta^{\prime}\right|\right] \rightarrow 0 \quad(j \rightarrow+\infty)
$$


Proof. This is a folklore. The statement is in fact trivial in case that

$$
f\left(\zeta^{\prime}\right)=\exp \left[-i\left\langle z^{\prime 0}, \zeta^{\prime}\right\rangle\right]
$$

for some fixed $z^{\prime 0}$ with $\left|z^{\prime 0}\right| \leq \delta$. For

$\left|\left(f-f_{j}\right)\left(\zeta^{\prime}\right)\right|=\left|\sum_{|k|=j+1}^{\infty}\left(-i\left\langle z^{\prime 0}, \zeta^{\prime}\right\rangle\right)^{k} / k !\right| \leq \sum_{|k|=j+1}^{\infty}\left|\delta \zeta^{\prime}\right|^{k} / k ! \leq\left(\delta / \delta^{\prime}\right)^{j} \exp \left[\delta^{\prime}\left|\zeta^{\prime}\right|\right]$.

From this observation, the statement follows for general $f$ if we write $f$ in the form

$$
f\left(\zeta^{\prime}\right)=\hat{\mu}\left(\zeta^{\prime}\right):=\int_{\mathbb{C}^{d}} \exp \left(-i\left\langle z^{\prime}, \zeta^{\prime}\right\rangle\right) d \lambda\left(z^{\prime}\right)
$$

by using a continuous function $\mu$ with support in $\left\{\left|z^{\prime}\right|<\left(\delta+\delta^{\prime}\right) / 2\right\}$. We can then argue as before with $f_{j}$ replaced by $f_{j}\left(\zeta^{\prime}\right)=\int_{\mathbb{C}^{d}} \sum_{|k| \leq j}\left[\left(-i\left\langle z^{\prime}, \zeta^{\prime}\right\rangle\right)^{k} /\right.$ $k !] \mu\left(z^{\prime}\right) d \lambda\left(z^{\prime}\right)$. (One can prove the proposition in another way using the Cauchy integral formula and Stirling's formula.)

Proposition 3.3. Let $0<\delta<\delta^{\prime}$ be given and assume $f \in \mathcal{O}_{\mathbb{C}^{d}}\left(\mathbb{C}^{d}\right)$ satisfies $\left|f\left(\zeta^{\prime}\right)\right| \leq \exp \left[H_{U^{\prime}+B^{\prime}(\delta)}\left(\zeta^{\prime}\right)\right]$. Then we can find $c, z^{\prime 1}, \ldots, z^{\prime s} \in U^{\prime}$, and $f_{k} \in \mathcal{O}_{\mathbb{C}^{d}}\left(\mathbb{C}^{d}\right)$ so that $f=\sum_{k=1}^{s} f_{k}$,

$$
\left|f_{k}\left(\zeta^{\prime}\right)\right| \leq c \exp \left[-\operatorname{Re}\left(i\left\langle z^{\prime k}, \zeta^{\prime}\right\rangle\right)+\delta^{\prime}\left|\zeta^{\prime}\right|\right]
$$

In the sequel we shall denote by $|f|_{A^{\prime}}$ for $A^{\prime} \subset \mathbb{C}^{d}$ the quantity:

$$
|f|_{A^{\prime}}=\sup _{\zeta^{\prime} \in \mathbb{C}^{d}}\left|f\left(\zeta^{\prime}\right)\right| / \exp \left[H_{A^{\prime}}\left(\zeta^{\prime}\right)\right]
$$

Combining Propositions 3.2 and 3.3, we now obtain:

Proposition 3.4. Let $0<\delta<\delta^{\prime}$ be given. Assume that $f \in \mathcal{O}_{\mathbb{C}^{d}}\left(\mathbb{C}^{d}\right)$ satisfies $\left|f\left(\zeta^{\prime}\right)\right| \leq \exp \left[H_{U^{\prime}+B^{\prime}(\delta)}\left(\zeta^{\prime}\right)\right]$. Then there is a sequence $f_{j} \in \mathcal{O}_{\mathbb{C}^{d}}\left(\mathbb{C}^{d}\right)$ such that

$$
\sup _{\zeta^{\prime}}\left|f\left(\zeta^{\prime}\right)-f_{j}\left(\zeta^{\prime}\right)\right| / \exp \left[H_{U^{\prime}+B^{\prime}\left(\delta^{\prime}\right)}\left(\zeta^{\prime}\right)\right] \rightarrow 0
$$

and for which

$$
\left|f_{j}\right|_{U^{\prime}}<\infty
$$


If we take into account of the characterization of analytic functionals by their Fourier-Borel transform, this proposition is considered to be as a quantitative version of the elementary fact that analytic functionals on $U^{\prime}+B^{\prime}\left(\delta^{\prime}\right)$ can be approximated in $\mathcal{O}^{\prime}\left(U^{\prime}+B^{\prime}\left(\delta^{\prime}\right)\right)$ by analytic functionals supported by $U^{\prime}$.

Proof of Theorem 3.1. We consider $z^{\prime} \in U^{\prime}+B^{\prime}(\delta)$, and fix some $\tilde{U}^{\prime}, \delta^{\prime}$ and $\delta^{\prime \prime}$ satisfying the conditions $\tilde{U}^{\prime} \subset \subset U^{\prime}, \delta^{\prime \prime}<\delta^{\prime}<\delta$ and $z^{\prime} \in \tilde{U}^{\prime}+B^{\prime}\left(\delta^{\prime \prime}\right)$. Thus $\delta_{z^{\prime}} \in \mathcal{O}_{\mathbb{C}^{d}}^{\prime}\left(\tilde{U}^{\prime}+B^{\prime}\left(\delta^{\prime \prime}\right)\right)$ and we can find a sequence $f_{j} \in \mathcal{O}_{\mathbb{C}^{d}}\left(\mathbb{C}^{d}\right)$ by Proposition 3.4 so that $\left|f_{j}\left(\zeta^{\prime}\right)\right| \leq C_{j} \exp \left[H_{\tilde{U}^{\prime}}\left(\zeta^{\prime}\right)\right],\left|f-f_{j}\right|_{U^{\prime}+B^{\prime}\left(\delta^{\prime}\right)} \rightarrow 0$ for $f=\hat{\delta}_{z^{\prime}}$. In particular, $\mathcal{F}^{-1} f_{j} \in \mathcal{O}_{\mathbb{C}^{d}}^{\prime}\left(U^{\prime}\right)$. We shall then define a function $H$ by setting $H\left(z^{\prime}\right)=\lim _{j \rightarrow \infty}\left(\mathcal{F}^{-1} f_{j}\right)(h)$. (The fact that the limit exists is immediate in that $f_{j}$ is a Cauchy-sequence in the norm $|\cdot|_{U^{\prime}+B^{\prime}\left(\delta^{\prime}\right)}$, so that also $\left(\mathcal{F}^{-1} f_{j}\right)(h)$ must be a Cauchy-sequence in view of (3.2).) The proof will come to an end if we can show that $H$ is well-defined, holomorphic, and extends $h$. The fact that $H$ is well-defined means that the definition of $H$ does not depend on the choice of the sequence $f_{j}$ associated with $f=\hat{\delta}_{z^{\prime}}$ above. This is clearly a consequence of the assumptions on $h$, in that if $f_{j}$ and $f_{j}^{\prime}$ are both in $\mathcal{O}_{\mathbb{C}^{d}}\left(\mathbb{C}^{d}\right)$ and satisfy $\left|f_{j}-f_{j}^{\prime}\right|_{U^{\prime}+B^{\prime}\left(\delta^{\prime}\right)} \rightarrow 0, \mathcal{F}^{-1} f_{j}, \mathcal{F}^{-1} f_{j}^{\prime} \in \mathcal{O}_{\mathbb{C}^{d}}^{\prime}\left(U^{\prime}\right)$, then $\mathcal{F}^{-1}\left(f_{j}-f_{j}^{\prime}\right)(h) \rightarrow 0$. The fact that $H$ is well-defined also proves that $H$ is an extension of $h$. As for the fact that $H$ is analytic, we first notice that the same construction defines $H$ as a functional on $\mathcal{O}_{\mathbb{C}^{d}}^{\prime}\left(U^{\prime}+B^{\prime}(\delta)\right)$ and that as such $H\left(\left(\partial / \partial \bar{z}_{k}^{\prime}\right) \delta_{z^{\prime}}\right)$ is the limit of $H\left(\left(\partial / \partial \bar{z}_{k}^{\prime}\right) \mathcal{F}^{-1} f_{j}\right)=0$, where the $f_{j}$ approximate $\exp \left[-i\left\langle z^{\prime}, \zeta^{\prime}\right\rangle\right]$ as before.

3. We next prove a modified version of Hartogs' theorem. We only consider the case of convex sets.

Theorem 3.5 (Hartogs-type theorem). Let $U^{\prime} \subset \mathbb{C}^{d}$ and $U^{\prime \prime} \subset \mathbb{C}^{n-d}$ be bounded open convex domains with $0 \in \partial U^{\prime}, U^{\prime \prime} \cap \mathbb{R}^{n-d} \neq \emptyset$ and $h \in$ $\mathcal{O}_{\mathbb{C}^{n}}\left(U^{\prime} \times U^{\prime \prime}\right)$ a holomorphic function defined on $U^{\prime} \times U^{\prime \prime}$. Assume that for any $x^{\prime \prime} \in U^{\prime \prime} \cap \mathbb{R}^{n-d}$, there exists a positive number $\delta\left(x^{\prime \prime}\right)>0$ for which the function $h\left(\cdot, x^{\prime \prime}\right) \in \mathcal{O}_{\mathbb{C}^{d}}\left(U^{\prime}\right)$ extends holomorphically to $B^{\prime}\left(\delta\left(x^{\prime \prime}\right)\right)$. Then we can find an open ball $B^{\prime \prime} \subset U^{\prime \prime}$ centered at some point $\dot{x}^{\prime \prime} \in U^{\prime \prime} \cap \mathbb{R}^{n-d}$ and a constant $\delta>0$ in such a way that $h$ extends holomorphically to $B^{\prime}(\delta) \times B^{\prime \prime}$.

Before we enter the proof of Theorem 3.5, we recall a local variant of the Phragmén-Lindelöf principle.

Lemma 3.6. Let $B^{\prime \prime}$ be the unit disc in $\mathbb{C}^{n-d}$ and let $\rho: B^{\prime \prime} \rightarrow \mathbb{R}$ be a plurisubharmonic function on $B^{\prime \prime}$. Assume that $\rho\left(z^{\prime \prime}\right) \leq 1$ on $B^{\prime \prime}$ and that 
$\rho\left(x^{\prime \prime}\right) \leq 0$ for $x^{\prime \prime} \in \mathbb{R}^{n-d} \cap B^{\prime \prime}$. Then there is a constant $C$ independent of $\rho$ satisfying $\rho\left(z^{\prime \prime}\right) \leq C\left|\operatorname{Im} z^{\prime \prime}\right|$ for $\left|z^{\prime \prime}\right| \leq 1 / 2$.

For a proof of this result cf. e.g. Meise-Taylor-Vogt [9]. Note that the lemma implies in a trivial way the following remark:

Remark 3.7. Assume that $\rho: B^{\prime \prime}(\varepsilon) \rightarrow \mathbb{R}$ is plurisubharmonic but assume now that $\rho\left(z^{\prime \prime}\right) \leq c$ on $B^{\prime \prime}(\varepsilon)$ whereas $\rho\left(x^{\prime \prime}\right) \leq c^{\prime}$ for $x^{\prime \prime} \in \mathbb{R}^{n-d} \cap B^{\prime}(\varepsilon)$ for some constants $c, c^{\prime}$. Then $\rho\left(z^{\prime \prime}\right) \leq c^{\prime}+c C\left|\operatorname{Im} z^{\prime \prime}\right|$ for $\left|z^{\prime \prime}\right| \leq \varepsilon / 2$. In particular it follows that if we fix $c^{\prime \prime}>c^{\prime}$ that there is $\varepsilon^{\prime}$ (which depends on $c$ and $C$ but not on $\rho$ ) so that $\rho\left(z^{\prime \prime}\right) \leq c^{\prime \prime}$ if $\left|z^{\prime \prime}\right|<\varepsilon^{\prime}$.

Proof of Theorem 3.5. Take any compact convex set $K^{\prime} \subset \subset U^{\prime}$ whose interior Int $K^{\prime}$ is non-empty and denote by $\hat{K}^{\prime}$ the convex hull of the set $\{0\} \cup K^{\prime}$ in $\mathbb{C}^{d}$. Then we have:

- $0 \in \partial \hat{K}^{\prime}$,

- $h$ is holomorphic in $\operatorname{Int} \hat{K}^{\prime} \times U^{\prime \prime}$,

- for any $x^{\prime \prime} \in U^{\prime \prime} \cap \mathbb{R}^{n-d}, h\left(\cdot, x^{\prime \prime}\right)$ extends holomorphically to a neighborhood of $\hat{K}^{\prime}$.

Thus by shrinking $U^{\prime}$ to Int $\hat{K}^{\prime}$ and by also shrinking $\delta\left(x^{\prime \prime}\right)$ suitably, we may assume, from the beginning, that for any $x^{\prime \prime} \in U^{\prime \prime} \cap \mathbb{R}^{n-d}$, the function $h\left(\cdot, x^{\prime \prime}\right)$ extends holomorphically to $U^{\prime}+B^{\prime}\left(\delta\left(x^{\prime \prime}\right)\right)$.

Set

$$
E_{j}^{\prime \prime}:=\bigcap_{v \in \mathcal{O}_{\mathbb{C}^{d}}^{\prime}\left(U^{\prime}\right),\left|\hat{v}\left(\zeta^{\prime}\right)\right| \leq \exp H_{U^{\prime}+B^{\prime}(1 / j)}\left(\zeta^{\prime}\right)}\left\{x^{\prime \prime} \in U^{\prime \prime} \cap \mathbb{R}^{n-d} ;\left|v\left(h\left(\cdot, x^{\prime \prime}\right)\right)\right| \leq j\right\} .
$$

By Theorem 3.1, we have that $U^{\prime \prime} \cap \mathbb{R}^{n-d}=\bigcup_{j} E_{j}^{\prime \prime}$. We can also see that every $E_{j}^{\prime \prime} \subset U^{\prime \prime} \cap \mathbb{R}^{n-d}$ is closed. Thus from Baire's principle, some $E_{j_{0}}^{\prime \prime}$ must include an open ball $E^{\prime \prime}:=\left\{x^{\prime \prime} \in \mathbb{R}^{n-d} ;\left|x^{\prime \prime}-\dot{x}^{\prime \prime}\right|<\varepsilon\right\}$. By shrinking $\varepsilon$, we can assume that $\dot{x}^{\prime \prime}+B^{\prime \prime}(\varepsilon) \subset \subset U^{\prime \prime}$. We define $\delta$ by $\delta=1 /\left(4 j_{0}\right)$, take a point $\dot{z}^{\prime} \in U^{\prime}$ with $\left|\dot{z}^{\prime}\right|<\delta$, and also take a positive constant $\delta^{\prime}$ with $\dot{z}^{\prime}+B^{\prime}\left(\delta^{\prime}\right) \subset \subset U^{\prime}$. From the considerations above, our function $h$ satisfies:

- $h$ is holomorphic in a neighborhood of the closure of $\left(\dot{z}^{\prime}+B^{\prime}\left(\delta^{\prime}\right)\right) \times\left(\dot{x}^{\prime \prime}+\right.$ $\left.B^{\prime \prime}(\varepsilon)\right)$, 
- each $h\left(\cdot, x^{\prime \prime}\right)$ satisfies $\left|v\left(h\left(\cdot, x^{\prime \prime}\right)\right)\right| \leq j_{0}$ for any $v \in \mathcal{O}_{\mathbb{C}^{d}}^{\prime}\left(\dot{z}^{\prime}+B^{\prime}(\delta)\right)$ with $\left|\hat{v}\left(\zeta^{\prime}\right)\right| \leq \exp H_{\dot{z}^{\prime}+B^{\prime}(3 \delta)}\left(\zeta^{\prime}\right)$.

It follows from the second property that for any $x^{\prime \prime} \in \mathbb{R}^{n-d}$ with $\left|x^{\prime \prime}-\dot{x}^{\prime \prime}\right|<$ $\varepsilon, h\left(\cdot, x^{\prime \prime}\right)$ extends holomorphically to $\dot{z}^{\prime}+B^{\prime}(3 \delta)$. This remark provides an alternative way to the result if we apply Lemma 4.4 .8 of A. Kaneko [3]. We give, however, the following proof to make this article more transparent.

Take the Taylor expansion of $h$ in the variables $z^{\prime}$ around $\dot{z}^{\prime}$ :

$$
h(z)=\sum_{\alpha} a_{\alpha}\left(z^{\prime \prime}\right)\left(z^{\prime}-\dot{z}^{\prime}\right)^{\alpha} .
$$

Now we will estimate the functions $z^{\prime \prime} \rightarrow\left|a_{\alpha}\left(z^{\prime \prime}\right)\right|$ in two ways. Our aim is to show that $a_{\alpha}\left(z^{\prime \prime}\right)$ satisfy estimates which are good enough to ensure that the function $h$ is analytic on a larger domain than its initial domain of definition.

First we use Cauchy's integral formula in the variables $z^{\prime}$ on the domain $\dot{z}^{\prime}+B^{\prime}\left(\delta^{\prime}\right)$ and obtain:

$$
\left|a_{\alpha}\left(z^{\prime \prime}\right)\right| \leq C_{1} \delta^{-|\alpha|} \quad \text { for any } z^{\prime \prime} \in \dot{x}^{\prime \prime}+B^{\prime \prime}(\varepsilon) \text { and any } \alpha,
$$

where $C_{1}:=\sup _{z \in\left(\dot{z}^{\prime}+B^{\prime}\left(\delta^{\prime}\right)\right) \times\left(\dot{x}^{\prime \prime}+B^{\prime \prime}(\varepsilon)\right)}|h(z)|$.

On the other hand, a stronger estimate can be obtained for the $a_{\alpha}\left(z^{\prime \prime}\right)$ for real arguments. Here we start from the expression:

$$
a_{\alpha}\left(z^{\prime \prime}\right)=v_{\alpha}\left(h\left(\cdot, z^{\prime \prime}\right)\right),
$$

where $v_{\alpha}$ is the analytic functional $v_{\alpha}: f \mapsto v_{\alpha}(f):=\left.(1 / \alpha !)\left(\left(\partial / \partial z^{\prime}\right)^{\alpha} f\right)\right|_{z^{\prime}=\dot{z}^{\prime}}$. Hence it follows that $\hat{v}_{\alpha}\left(\zeta^{\prime}\right)=(1 / \alpha !)\left(-i \zeta^{\prime}\right)^{\alpha} \exp \left(-i\left\langle\dot{z}^{\prime}, \zeta^{\prime}\right\rangle\right)$ and that

$$
\sup _{\zeta^{\prime} \in \mathbb{C}^{d}}\left|\hat{v}_{\alpha}\left(\zeta^{\prime}\right)\right| \exp \left(-H_{\dot{z}^{\prime}+B^{\prime}(3 \delta)}\left(\zeta^{\prime}\right)\right)=\frac{1}{\alpha !} \prod_{j=1}^{d} \sup _{t \geq 0} t^{\alpha_{j}} \exp (-3 \delta t)=\frac{\alpha^{\alpha}}{\alpha !(3 \delta e)^{|\alpha|}}
$$

(using $k !>\exp \int_{1}^{k} \log x d x=k^{k} e^{1-k}$ )

$$
\leq \frac{1}{e^{d}(3 \delta)^{|\alpha|}}
$$

These considerations show that

$$
\left|a_{\alpha}\left(x^{\prime \prime}\right)\right| \leq C_{2}(3 \delta)^{-|\alpha|} \quad \text { for any } x^{\prime \prime} \in \mathbb{R}^{n-d} \text { with }\left|x^{\prime \prime}-\dot{x}^{\prime \prime}\right|<\varepsilon \text { and any } \alpha,
$$


where $C_{2}:=j_{0} e^{-d}$.

In this situation we apply Remark 3.7 to the plurisubharmonic functions

$$
z^{\prime \prime} \rightarrow u_{\alpha}\left(z^{\prime \prime}\right)=\frac{1}{|\alpha|}\left(\log \left|a_{\alpha}\left(z^{\prime \prime}+\dot{x}^{\prime \prime}\right)\right|-\log C\right),
$$

where $C=\max \left(C_{1}, C_{2}\right)$. The estimates (3.8) and (3.9) proved above show that

$$
\begin{aligned}
& u_{\alpha}\left(z^{\prime \prime}\right) \leq-\log \delta^{\prime} \quad \text { on } B^{\prime \prime}(\varepsilon), \\
& u_{\alpha}\left(x^{\prime \prime}\right) \leq-\log (3 \delta) \quad \text { on } B^{\prime \prime}(\varepsilon) \cap \mathbb{R}^{n-d} .
\end{aligned}
$$

We can now apply Remark 3.7 to obtain that

$$
u_{\alpha}\left(z^{\prime \prime}\right) \leq-\log (2 \delta) \quad \text { on } B^{\prime \prime}\left(\varepsilon^{\prime}\right),
$$

with some constant $\varepsilon^{\prime}$. We have therefore obtained the estimate

$$
\left|a_{\alpha}\left(z^{\prime \prime}\right)\right| \leq C(2 \delta)^{-|\alpha|} \quad \text { for any } z^{\prime \prime} \in \dot{x}^{\prime \prime}+B^{\prime \prime}\left(\varepsilon^{\prime}\right) .
$$

This estimate shows that for any $z^{\prime \prime} \in \dot{x}^{\prime \prime}+B^{\prime \prime}\left(\varepsilon^{\prime}\right)$, the Taylor series (3.7) converges at least on $\dot{z}^{\prime}+B^{\prime}(2 \delta)$ and that our function $h$ extends holomorphically to the domain $\left(\dot{z}^{\prime}+B^{\prime}(2 \delta)\right) \times\left(\dot{x}^{\prime \prime}+B^{\prime \prime}\left(\varepsilon^{\prime}\right)\right)$, which includes $B^{\prime}(\delta) \times\left(\dot{x}^{\prime \prime}+B^{\prime \prime}\left(\varepsilon^{\prime}\right)\right)$. Thus we have the desired result if we take $\dot{x}^{\prime \prime}+B^{\prime \prime}\left(\varepsilon^{\prime}\right)$ for $B^{\prime \prime}$.

4. Now we give a proof of Theorem 2.2.

Proof of Theorem 2.2. Let us then assume that $h$ is a holomorphic function satisfying the assumption of Theorem 2.2. For each $\dot{x}^{\prime \prime} \in \mathbb{R}^{n-d}$ with $\left|\dot{x}^{\prime \prime}\right|<\varepsilon$, the restriction $h\left(\cdot, \dot{x}^{\prime \prime}\right)$ extends holomorphically to a set of type $\left\{z^{\prime} ;\left|z^{\prime}\right|<\delta\left(\dot{x}^{\prime \prime}\right)\right\}$ with some positive number $\delta\left(\dot{x}^{\prime \prime}\right)$.

Then from Theorem 3.5, we can take a positive constant $\delta$ and an open ball $B^{\prime \prime} \subset\left\{z^{\prime \prime} \in \mathbb{C}^{n-d} ;\left|z^{\prime \prime}\right|<\varepsilon\right\}$ centered at some point $\dot{x}^{\prime \prime} \in \mathbb{R}^{n-d}$ such that $h$ extends holomorphically to $B^{\prime}(\delta) \times B^{\prime \prime}$. Let us consider the boundary value $u=$ $\dot{b}(h) \in \mathcal{B O}\left(\left\{\left(x^{\prime}, z^{\prime \prime}\right) ;\left|x^{\prime}\right|<\varepsilon,\left|z^{\prime \prime}\right|<\varepsilon\right\}\right)$. From the domain of holomorphy of $h$, we can see that $u$ is real analytic on the domain $\left\{\left(x^{\prime}, z^{\prime \prime}\right) ;\left|x^{\prime}\right|<\delta, z^{\prime \prime} \in B^{\prime \prime}\right\}$. Then from the unique continuation property for the analytic wave front sets along holomorphic parameters, we can conclude that $u$ is real analytic on the domain $\left\{\left(x^{\prime}, z^{\prime \prime}\right) ;\left|x^{\prime}\right|<\delta,\left|z^{\prime \prime}\right|<\varepsilon\right\}$. Since there is only one defining function $h$ in the boundary value representation $u=\dot{b}(h)$, the real analyticity of $u$ asserts that $h$ extends holomorphically to this domain $\left\{\left(x^{\prime}, z^{\prime \prime}\right) ;\left|x^{\prime}\right|<\delta,\left|z^{\prime \prime}\right|<\varepsilon\right\}$. This completes the proof. 


\section{$\S 4$. Proof of Theorem 1.6}

We shall show that Theorem 1.6 follows from Theorem 2.2. We may assume that $M^{\prime}=\mathbb{R}^{d}, X^{\prime \prime}=\mathbb{C}^{n-d}, 0 \in \Omega^{\prime}, 0 \in U^{\prime \prime} \cap M^{\prime \prime}$, and that $\dot{q}^{\prime}=\left(0 ; d x_{1}\right)$. By shrinking $\Omega^{\prime}$ to a sufficiently small neighborhood of $0=\dot{\pi}_{M^{\prime}}\left(\dot{q}^{\prime}\right)$, we may assume that

$$
Z \subset\left\{\left(x^{\prime}, \xi^{\prime}\right) ; \xi_{1}>0\right\}
$$

From the assumptions (1.6) and (4.1), it is easy to see that $u$ admits a representation

$$
u=\dot{b}(h) \text { on }\left\{\left(x^{\prime}, z^{\prime \prime} ; \xi^{\prime}\right) ;\left|x^{\prime}\right|<\varepsilon,\left|z^{\prime \prime}\right|<\varepsilon, \xi^{\prime} \neq 0\right\}
$$

for some small $\varepsilon>0$, some holomorphic function

$$
h(z) \in \mathcal{O}\left(\left\{z ;\left|z^{\prime}\right|<\varepsilon, y^{\prime} \in G^{\prime},\left|z^{\prime \prime}\right|<\varepsilon\right\}\right)
$$

and some conic neighborhood $G^{\prime} \subset \mathbb{R}^{d} \backslash\{0\}$ of $(1,0, \ldots, 0)$.

From the assumption $u_{\mid z^{\prime \prime}=\dot{x}^{\prime \prime}}=b\left(h\left(\cdot, \dot{x}^{\prime \prime}\right)\right)=0$ at $\dot{q}^{\prime}$ for any $\dot{x}^{\prime \prime} \in U^{\prime \prime} \cap M^{\prime \prime}$, it is immediate that $h\left(\cdot, \dot{x}^{\prime \prime}\right)=h_{\mid z^{\prime \prime}=\dot{x}^{\prime \prime}}$ extends holomorphically to a neighborhood of $0 \in \mathbb{C}^{d}$, for every $\dot{x}^{\prime \prime} \in \mathbb{R}^{d}$ with $\left|\dot{x}^{\prime \prime}\right|<\varepsilon$. Thus we can deduce that $h$ satisfies all the assumptions of Theorem 2.2. It follows therefore from that theorem that $\dot{b}(h)$ is 0 as a microfunction with holomorphic parameters on $\left\{\left(x^{\prime}, z^{\prime \prime} ; \xi^{\prime}\right) ; x^{\prime}=0,\left|z^{\prime \prime}\right|<\varepsilon, \xi^{\prime} \neq 0\right\}$. In particular $u=0$ in a neighborhood of $\left\{\dot{q}^{\prime}\right\} \times\left\{z^{\prime \prime} ;\left|z^{\prime \prime}\right|<\varepsilon\right\}$. This gives the desired result in view of the unique continuation property for microfunctions with holomorphic parameters.

\section{$\S 5 . \quad$ Proof of Theorem 1.4}

1. In this section we will reduce Theorem 1.4 to Theorem 1.6. Since we have already proved Theorem 1.6, this will conclude the proof of all results mentioned in the introduction. The argument is based on two morphisms due to Kashiwara and which were used in the proof of the flabbiness of the sheaf of microfunctions.

Remark 5.1. Actually, it is possible to avoid the use of the Kashiwara morphisms in the proof of Theorem 1.4. The basic idea is to use Baire's theorem in combination with a microlocal characterization of $\mathrm{WF}_{A}$ by duality (based on O. Liess [7], [8]), rather than in combination with Theorem 3.1. This leads to a proof in which one obtains directly Theorem 1.4, without passing through 1.6 . 
To turn to the proofs, we first observe that it suffices to prove the Theorem 1.4 in the case when $\dot{q}^{\prime} \notin M^{\prime}$.

In fact, in case of $\dot{q}^{\prime} \in M^{\prime}$, we may assume that $u$ is defined in a neighborhood of $\pi_{M^{\prime}}^{-1}\left(\dot{q}^{\prime}\right) \times U^{\prime \prime}$ since $\mathcal{C} \mathcal{O}_{N}$ is conic. For any point $q^{\prime} \in \dot{\pi}_{M^{\prime}}^{-1}\left(\dot{q}^{\prime}\right)$ we can apply the theorem for $u$ and $q^{\prime}$, and get $u=0$ in a neighborhood of $\dot{\pi}_{M^{\prime}}^{-1}\left(\dot{q}^{\prime}\right) \times U^{\prime \prime}$. This means that $u$ is real analytic in a neighborhood of $\left\{\dot{q}^{\prime}\right\} \times U^{\prime \prime}$, and we get the desired result from the unique continuation property of real analytic functions.

2. Now we concentrate on the case when $\dot{q}^{\prime} \notin M^{\prime}$. Since the sheaf of microfunctions with holomorphic parameter is conic, we may work on conormal sphere bundles instead of conormal bundles.

Let $S_{M^{\prime}}^{*} X^{\prime}:=\dot{T}_{M^{\prime}}^{*} X^{\prime} / \mathbb{R}_{+}$be the conormal sphere bundle along $M^{\prime}$ and $\gamma^{\prime}: \dot{T}_{M^{\prime}}^{*} X^{\prime} \rightarrow S_{M^{\prime}}^{*} X^{\prime}$ the canonical projection. We use the identifications $S_{M^{\prime}}^{*} X^{\prime}=\sqrt{-1} S^{*} M^{\prime} \simeq S^{*} M^{\prime}$ and $S_{N}^{*} X \simeq S^{*} M^{\prime} \times X^{\prime \prime}$, and the convention $\gamma=\gamma^{\prime} \times \operatorname{id}_{X^{\prime \prime}}$.

In this section, we denote the sheaves $\gamma_{*}^{\prime} \mathcal{C}_{M^{\prime}}, \gamma_{*} \mathcal{C O}_{N}$ by $\mathcal{C}_{M^{\prime}}^{\prime}$, respectively by $\mathcal{C O}_{N}^{\prime}$.

Now we recall the Kashiwara morphisms briefly.

Let $L:=\mathbb{R}^{d}$ and $Y:=S^{*} L \simeq \mathbb{R}^{d} \times S^{d-1}$, regarded as real analytic manifolds with coordinates $x^{\prime}$ and $\left(x^{\prime}, \xi^{\prime}\right)$ respectively. We define two closed subsets $Z$ and $Z_{0}$ in $S^{*} Y$ by

$$
Z=\left\{\left(x^{\prime}, \xi^{\prime} ; \xi^{\prime} \cdot d x^{\prime}\right)\right\}, \quad Z_{0}=\left\{\left(0, \xi^{\prime} ; \xi^{\prime} \cdot d x^{\prime}\right)\right\}
$$

We denote by $\pi_{Y}$ the canonical projection $S^{*} Y \rightarrow Y$.

We cite the following result. (Refer to Sato-Kawai-Kashiwara [11, Chap. III, Corollary 2.1.5, p.473] or Kashiwara-Kawai-Kimura [4, Chap. III, Theorem 3.7.1]).

Lemma 5.2. There exist two microfunctions $K\left(x^{\prime}, \xi^{\prime}\right), T\left(x^{\prime}, \xi^{\prime}\right) \in$ $\mathcal{C}_{Y}^{\prime}\left(S^{*} Y\right)$, such that

$$
\operatorname{supp} K \subset Z_{0}, \quad \operatorname{supp} T \subset Z_{0},
$$

and that

$$
\int_{S^{d-1}} \int_{\mathbb{R}^{d}} T\left(x^{\prime}-y^{\prime}, \xi^{\prime}\right) K\left(y^{\prime}, \xi^{\prime}\right) d y^{\prime} \omega\left(\xi^{\prime}\right)=\delta\left(x^{\prime}\right) .
$$

(We have denoted by $\omega$ the volume element on $S^{d-1}$.) 
Using these microfunctions, we can define the following sheaf morphisms.

Lemma 5.3. Let $U^{\prime} \subset Y$ and $U^{\prime \prime} \subset X^{\prime \prime}$ be two open subsets. Here $X^{\prime \prime}$ denotes an arbitrary complex manifold with local coordinates $z^{\prime \prime}$.

(1) For a section $u\left(x^{\prime}, z^{\prime \prime}\right) \in \mathcal{C O}_{L \times X^{\prime \prime}}^{\prime}\left(U^{\prime} \times U^{\prime \prime}\right)$, the integral

$$
\int K\left(x^{\prime}-y^{\prime}, \xi^{\prime}\right) u\left(y^{\prime}, z^{\prime \prime}\right) d y^{\prime}
$$

becomes a section of $\Gamma_{Z \times X^{\prime \prime}}\left(\pi_{Y}^{-1}\left(U^{\prime}\right) \times U^{\prime \prime}, \mathcal{C} \mathcal{O}_{Y \times X^{\prime \prime}}^{\prime}\right)$. This defines a sheaf morphism

$$
\Phi: \mathcal{C O}_{L \times X^{\prime \prime}}^{\prime} \rightarrow \pi_{Y *} \Gamma_{Z \times X^{\prime \prime}} \mathcal{C O}_{Y \times X^{\prime \prime}}^{\prime}
$$

(2) For a section $v\left(x^{\prime}, \xi^{\prime}, z^{\prime \prime}\right) \in \Gamma_{Z \times X^{\prime \prime}}\left(\pi_{Y}^{-1}\left(U^{\prime}\right) \times U^{\prime \prime}, \mathcal{C} \mathcal{O}_{Y \times X^{\prime \prime}}^{\prime}\right)$, the integral

$$
\int T\left(x^{\prime}-y^{\prime}, \xi^{\prime}\right) v\left(y^{\prime}, \xi^{\prime}, z^{\prime \prime}\right) d y^{\prime} \omega\left(\xi^{\prime}\right)
$$

becomes a section of $\mathcal{C O}_{L \times X^{\prime \prime}}^{\prime}\left(U^{\prime} \times U^{\prime \prime}\right)$. This defines a sheaf morphism

$$
\Psi: \pi_{Y *} \Gamma_{Z \times X^{\prime \prime}} \mathcal{C} \mathcal{O}_{Y \times X^{\prime \prime}}^{\prime} \rightarrow \mathcal{C O}_{L \times X^{\prime \prime}}^{\prime}
$$

(3) The morphisms $\Phi$ and $\Psi$ satisfy that

$$
\Psi \circ \Phi=\mathrm{id}
$$

(4) The morphisms $\Phi$ and $\Psi$ commute with the restriction $\left.\cdot\right|_{z^{\prime \prime}=\dot{z}^{\prime \prime}}$ with respect to $z^{\prime \prime}$ variable.

3. Finally we prove Theorem 1.4 assuming that Theorem 1.6 is already known to be true. Since the Theorem 1.4 is of a local nature, we may assume that the manifold $M^{\prime}$ in the theorem is $L=\mathbb{R}^{d}$. We will identify sections of $\mathcal{C} \mathcal{O}_{L \times X^{\prime \prime}}$ defined outside of $L \times X^{\prime \prime}$ with those of $\mathcal{C} \mathcal{O}_{L \times X^{\prime \prime}}^{\prime}$. We also identify $\dot{q}^{\prime}$ with $\gamma^{\prime}\left(\dot{q}^{\prime}\right)$. Let $u$ be a section of $\mathcal{C} \mathcal{O}_{L \times X^{\prime \prime}}$ satisfying the assumptions in the theorem.

From the assumption, $\left.u\right|_{z^{\prime \prime}=\dot{x}^{\prime \prime}}=0$ at $\dot{q}^{\prime}$ for any $\dot{x}^{\prime \prime} \in U^{\prime \prime} \cap M^{\prime \prime}$. Then from Lemma $5.3(4),\left.\Phi(u)\right|_{z^{\prime \prime}=\dot{x}^{\prime \prime}}$ is also 0 at $\pi_{Y}^{-1}\left(\dot{q}^{\prime}\right)$. Thus we can apply Theorem 1.6 to $\Phi(u)$ in case of $M^{\prime}=Y$ at a point $\pi_{Y}^{-1}\left(\dot{q}^{\prime}\right) \cap Z$ and get $\Phi(u)=0$ on $\pi_{Y}^{-1}\left(\dot{q}^{\prime}\right) \times U^{\prime \prime}$. Applying $\Psi$, we have $u=0$ in a neighborhood of $\left\{\dot{q}^{\prime}\right\} \times U^{\prime \prime}$. 


\section{References}

[1] Hörmander, L., An introduction to complex analysis in several variables, North-Holland Publ. Comp., Amsterdam-London, 3-rd revised printing, 1990.

[2] Kaneko, A., On hyperfunctions with real analytic parameters, Proc. of Workshop "Prospects of Algebraic Analysis", RIMS, Kyoto Univ. Kokyuroku, 675 (1988), 70-78 (in Japanese).

[3] - Introduction to hyperfunctions, Kluwer Academic Publishers, 1988.

[4] Kashiwara, M., Kawai, T. and Kimura, T., Foundations of algebraic analysis, Princeton University Press, Princeton, 1986.

[5] Kataoka, K., Private communication.

[6] Komatsu, H., A local version of Bochner's tube theorem, J. Fac. Sci. Univ. Tokyo, Sect IA, 19 (1972), 201-214.

[7] Liess, O., $\bar{\partial}$-cohomology with bounds and hyperfunctions, to be published.

[8] — - Higher microlocalization and propagation of singularities, Proceedings zu der Nato ASI über "Microlocal Analysis and Spectral Theory" 1996, Kluwer Academic publishers (1997), 61-91.

[9] Meise, R., Taylor, B. A. and Vogt, D., Phragmén-Lindelöf principles on algebraic varieties, J. Amer. Math. Soc., 11 (1998), 1-39.

[10] Sato, M., Theory of hyperfunctions I, J. Fac. Sci. Univ. Tokyo, Sect. I, 8 (1959), 139193; II, 8 (1960), 387-437.

[11] Sato, M., Kawai, T. and Kashiwara, M., Microfunctions and pseudo-differential equations, Hyperfunctions and pseudo-differential equations. Lecture Notes in Math., 287, Springer (1973), 265-529. 